\title{
POTENCIAL EFICACIA DE LA INOCULACION CON ENDOMICORRIZAS DEL GENERO Glomus EN MAIZ (Zea mays ) Y SU IMPACTO SOBRE LA ACTIVIDAD BIOLOGICA DEL SUELO*
}

\author{
(Potential effectiveness of the inoculation with mycorrhizae of the genus Glomus \\ in corn (Zea mays) and their impact on the biological activity of soil*)
}

\author{
Fogar Mariela N.; Iglesias María C.; Cracogna Mariano F. \\ Cátedra de Microbiología Agrícola. Facultad de Ciencias Agrarias. UNNE. \\ Sargento Cabral 2131. 3400 Corrientes, Argentina. \\ E mail: migles@agr.unne.edu.ar / mariaiglesias@ciudad.com.ar \\ *Trabajo realizado en el marco del convenio Cátedra de Microbiología \\ Agrícola - B.P.F. Argentina S.A.
}

Palabras clave: Micorrizas, maíz, suelo.

Key words: Mycorrhizae, corn, soil

\section{RESUMEN}

Los factores que afectan la respuesta de las plantas a la colonización por micorrizas, incluyen la dependencia del cultivo anfitrión de ésta, el estado de nutrientes del suelo, y el inóculo potencial de estos hongos. Las prácticas de manejo de cultivo, la rotación de ellos y el barbecho, pueden afectar a las poblaciones de hongos micorrícicos adversamente en el campo.

El objetivo de nuestro trabajo fue evaluar la respuesta a la inoculación con micorrizas no nativas en maíz para apreciar los efectos de la misma sobre la actividad biológica del suelo.

Se realizó un ensayo en macetas bajo invernáculo en un diseño en bloques completos al azar; con cuatro tratamientos y tres repeticiones. Los tratamientos corresponden a la inoculación con diferentes aislamientos de Glomus intraradices y Glomus mosseae.

Se analizaron las siguientes variables, \% de micorrización, peso seco total, Peso seco parte aérea y radical. En el suelo se realizó el análisis de amonificación, respiración, degradación de celulosa y presencia de Azotobacter sp .

Se concluye que la micorrización fue infectiva para los inóculos ensayados. No se reflejaron diferencias en el desarrollo de la planta. En relación con la actividad biológica del suelo, solo la presencia de Azotobacter fue diferente en algunos tratamientos.

\begin{abstract}
Factors that affect the colonization with mycorrhizae in the plants include the dependence of the host culture from the mycorrhizae, the state of nutrients in the soil, and the potential inoculum of the them. Practices such as the handling of cultivation its isolation as well as the fallow can adversely affect the populations of mycorrhizae in the field.

The objective of this works was to evaluate the response to the inoculation with non native mycorrhizae in corn, and its effect on the biological activity of the soil.

Complete blocks of flower pots kept under a greenhouse were tested at random, using four treatments and three repetitions. Treatments correspond to the inoculation with different isolations of Glomus intraradices and Glomus mosseae.

The following variables were analized: mycorrhizal percentage, total dry weight aerial and radical dry weight. The soil was analized as regards ammonia contents, breath, cellulose degradation and presence of Azotobacter sp.

It is concluded that the mycorrhizal was infective for the three inocula under test. No differences were detected in the development of the plant. As to the biological activity of the soil, only the presence of Azotobacter sp. showed different in some treatments.
\end{abstract}




\section{INTRODUCCION}

Las micorrizas arbusculares (MA) se encuentran en la mayoría de las plantas silvestres y cultivadas. Es bien conocido que aquellas que poseen micorrizas, pueden absorber nutrientes de su ambiente con más eficiencia que las que no las poseen. Es probable que esta mejora se deba a la mayor superficie que proporciona el micelio. El efecto beneficioso que se obtiene del hongo micorrícico se observa mejor en suelos de baja fertilidad. El fósforo es uno de los nutrientes que las MA transportan a través de sus hifas hacia las plantas (Miller \& Allen, 1992).

Para que la asociación micorrícica se produzca en plenitud, es necesario favorecer al hongo permitiendo que sus esporas colonicen las raíces de las plantas. Los implementos de labranza remueven al suelo y lo dejan expuesto a la luz solar y a la deshidratación, lo que termina por destruir las esporas del hongo y su ambiente, impidiendo en cierta medida su asociación (Crovetto, 2000).

Los factores que afectan la respuesta de las plantas a la colonización por hongos micorrícicos incluyen la dependencia del cultivo anfitrión de la micorriza, el estado de nutrientes del suelo y el inóculo potencial de estos hongos. Las prácticas de manejo de cultivo, su rotación y el barbecho pueden afectar a las poblaciones de hongos micorrícicos adversamente en el campo (Sylvia, 2000).

Las micorrizas obtienen por lo menos algunos de los azúcares de la planta, mientras la planta se beneficia de la captación de nutrientes minerales por la hifa fúngica. La influencia de las micorrizas sobre el consumo de nutrientes es bastante importante. Las raíces micorrizadas asimilan los fosfatos con mayor facilidad que las raíces sin hongos, permitiendo a la planta desarrollarse en suelos deficientes en este elemento. Además, el hongo estimula el consumo de nitrógeno, azufre y cinc entre otros, protegiendo simultáneamente a las raíces contra las infecciones provocadas por una gran variedad de agentes patógenos del suelo (Frioni, 1990,1999), por lo que puede ser utilizado en biocontrol (Linderman, 1994; Duchense, 1994).

La mejor forma de estimular esta acción simbiótica es dejar al suelo siempre protegido con cubierta vegetal, lo que se logra dejando de labrar y manejando eficientemente los rastrojos sobre él. Muchas veces es preferible ofrecer al suelo mejores condiciones ambientales que forzar su inoculación con individuos benéficos (Primavesi, 1994). Cuando el potencial de inoculo nativo es bajo o ineficaz, las estrategias de inoculación pueden ser útiles (Sylvia, 2000).

De lo expuesto, surgió el objetivo de evaluar la respuesta a la inoculación con micorrizas no nativas en maíz y estudiar su efecto en la actividad biológica del suelo.

\section{MATERIALES Y METODOS}

Mediante un ensayo en invernadero se usaron macetas con capacidad de $400 \mathrm{~g}$; en un diseño en bloques completos al azar con 4 tratamientos y tres repeticiones. Los tratamientos fueron:

1 - Testigo;

2 - Glomus intraradices Schenck \& Smith 1982 aislamiento SB1;

3 - Glomus mosseae (Nicolson \& Gerdemann) Gerdemann \& Trappe 1974, aislamiento Ne 42;

4 - Glomus mosseae (Nicolson \& Gerdemann) Gerdemann \& Trappe 1974, aislamiento SB2.

Todos los inóculos provienen del Instituto Spegazzini, La Plata Bs. As. Argentina.

El suelo utilizado fue un ustocrept údico, (serie tolosa), se encuentran en lomas tendidas, evolucionadas, de relieve normal, textura franca, con buen contenido de materia orgánica y pH neutro, que corresponde al Departamento Comandante Fernández (Chaco), colonia Domingo F. Sarmiento, chacra 131-Lote 5.

Se usó maíz (Zea mays L) como planta indicadora /trampa, las macetas fueron regadas periódicamente para mantener una humedad cercana a la capacidad de campo.

Se sembraron 5 semillas por maceta y la inoculación se realizó en el momento de la siembra. Al término de la experiencia, se procedió a extraer las plantas y acondicionar el suelo para las siguientes determinaciones.

\section{Porcentaje de micorrización:}

De cada maceta se extrajeron las plantas con raíces, se procedió al lavado de las raíces. Diez trozos al azar de cada muestra fueron tratados con azul de tripán, método de Phillips y Hayman, seguidamente se realizó la observación al microscopio con aumento de 600x calculándose los porcentajes de micorrización (Honrubia, 1995).

\section{Peso seco total (PST), Peso seco parte aérea (PSA) y radicular (PSR).}

Las plantas fueron secadas en estufa a $70{ }^{\circ} \mathrm{C}$ hasta peso constante. Luego se las pesó en balanza de precisión ( 0.001 g)

\section{Actividad amonificante:}

Se realizó por medio de incubación aeróbica del suelo, recogiendo el amoníaco producido en solución ácida y su valoración volumétrica con un álcali de norma- 
lidad conocida a los 7, 14 y 21 días (Frioni, 1990). Incubación controlada a $28-30^{\circ} \mathrm{C}$ de temperatura (Page et al., 1982).

\section{Capacidad de degradar celulosa:}

Se midió a los 7, 14 y 21 días, calculándose los porcentajes de masa remanente para cada tratamiento en cada fecha. Se utilizó la técnica de los microdiscos de papel de filtro en placas de suelo con una humedad del 60 $\%$ de la capacidad máxima de retención (Quant et al., 1981).

\section{Actividad respiratoria:}

Se realizó en el laboratorio mediante técnica de incubación, (Schinner et al., 1996). colocando $50 \mathrm{~cm}^{3}$ de material en bolsitas de polietileno permeables a gases e impermeables a vapor de agua, estas a su vez se introdujeron en frascos de cierre hermético. El dióxido de carbono producido se recogió en hidróxido de bario y se tituló con ácido oxálico y fenolftaleína como indicador (Fuentes \& Quant, 1971; Page et al., 1982).

\section{Presencia de fijadores libres de nitrógeno, Azotobacter sp.}

Se determinó mediante aparición espontánea, aplicando la Técnica Ecológica de la placa de tierra moldeada de Winogradsky, agregando las fuentes de carbono soluble necesarias para el género (Frioni, 1990).

Posteriormente los datos se analizaron mediante Analytical Software. El Análisis de la varianza, mediante Linear Models - General AOV/AOCV y prueba de Tukey $(\mathrm{P}<0,05)$ para la comparación de los promedios.

\section{RESULTADOS Y DISCUSION}

El porcentaje de micorrización presentó diferencias significativas estadísticamente, siendo el tratamiento 2 superior al testigo, pero no a los otros tratamientos (3 y 4). La presencia de infección en el testigo correspondería a MA nativas, dado que no hay referencias de anteriores inoculaciones en ese suelo.

Los tratamientos 3 y 4 no superaron al testigo estadísticamente, pero los valores registrados marcan una diferencia de consideración (Figura 1).También Covacevich et al. (2000), evaluando la receptividad de los suelos a inóculos de MA no nativas, manifestaron diferentes desarrollos de las mismas.

Para la variable promedio de peso seco parte aérea, no se encontró diferencias estadísticamente significativas, sin embargo, se puede observar que el tratamiento 4 presenta la media mas alta, mientras que el tratamiento 3 la media mas baja (Figura 2).

Con respecto al promedio de peso seco radical no

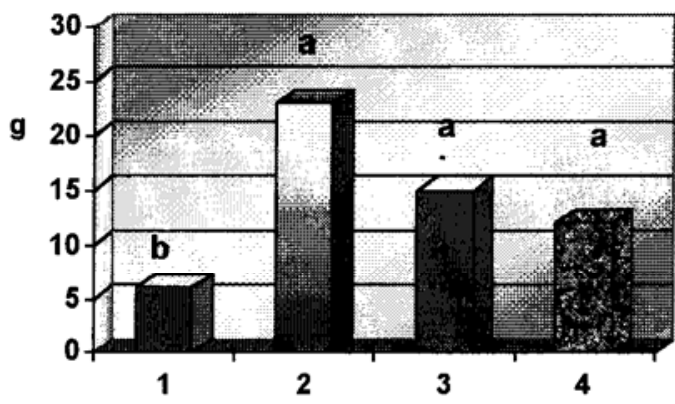

Figura 1.- Porcentaje de micorrización. * Letras iguales no se diferencian estadísticamente.

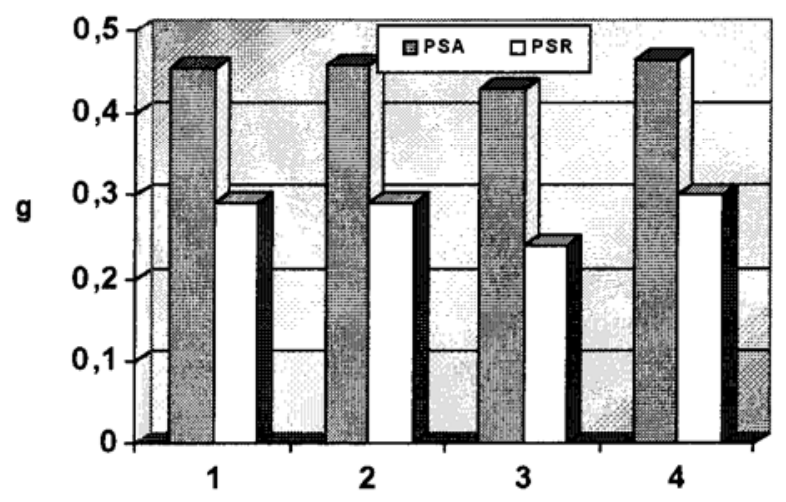

Figura 2.- Peso promedio de la parte aérea y radical por tratamientos.

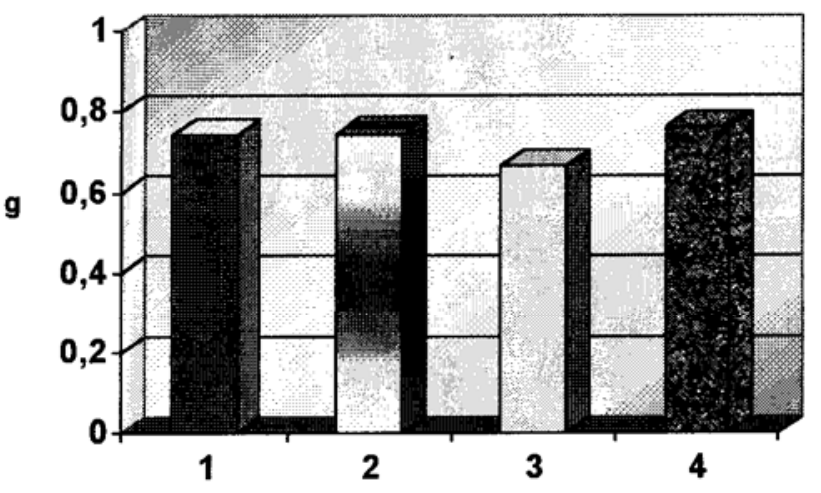

Figura 3.- Peso seco de la planta entera.

se obtuvo diferencias significativas estadísticamente, manteniéndose la relación observada en la parte aérea (Figura 2). La variable peso seco total, no mostró diferencias significativas, acentuándose las diferencias observadas en el análisis de las partes aérea y radical (Figura 3). Los resultados del desarrollo de las plantas no manifestaron las diferencias que se presentaron en los valores de infección micorrícica. Sin embargo, Nurlaeny et al. (1996), utilizando la inoculación con AM. Glomus intraradices en plantas de maíz en suelos tropicales, determinaron una alta infección y mayor peso seco tanto 


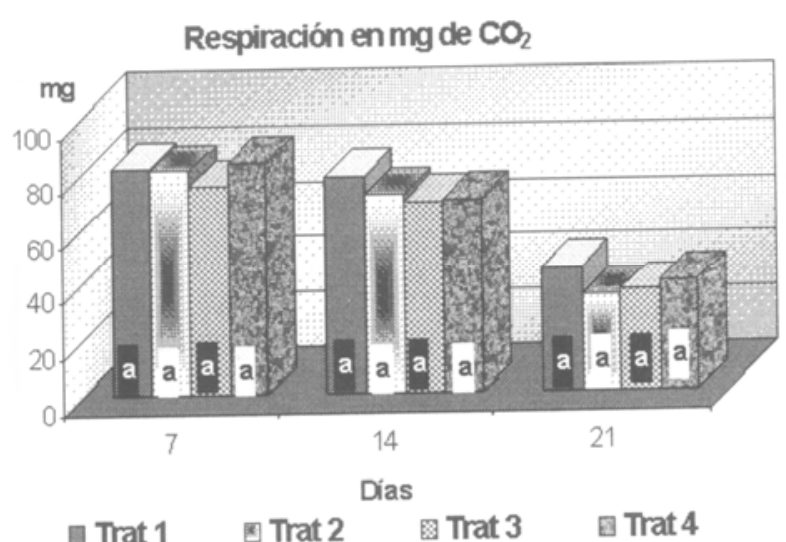

Figura 4.- Actividad respiratoria. * letras iguales no se diferencian estadísticamente.

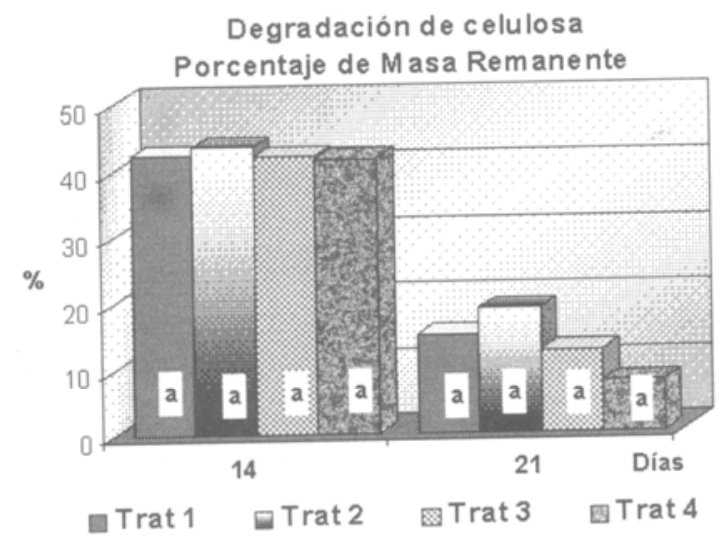

Figura 5: Actividad celulolítica. * Letras iguales no se diferencian estadísticamente.

en la parte aérea como radical.

Actividad Respiratoria.

En la figura 4 podemos observar la evolución de la respiración del suelo cada 7 días (7, 14 y 21 días) no encontrándose diferencias significativas en ninguno de los lapsos medidos. Sin embargo, el trat. 2 a los 7 días presentó un $0,81 \%$ menos en la tasa respiratoria, acentuándose la diferencia en las fechas siguientes con un $8,5 \%$ y un $22 \%$ menos respectivamente.

El trat. 3 marcó una mayor diferencia que el trat. 2 , en los primeros 7 días con respecto al testigo, con un $7,7 \%$ menos en la tasa respiratoria, incrementándose la diferencia a un $11,8 \%$ y un $18,7 \%$ menos para las dos fechas siguientes.

El trat 4 por su parte fue el único que presentó una mayor tasa respiratoria que el testigo, superándolo para la primera fecha en un $2,8 \%$, posteriormente bajó mar-

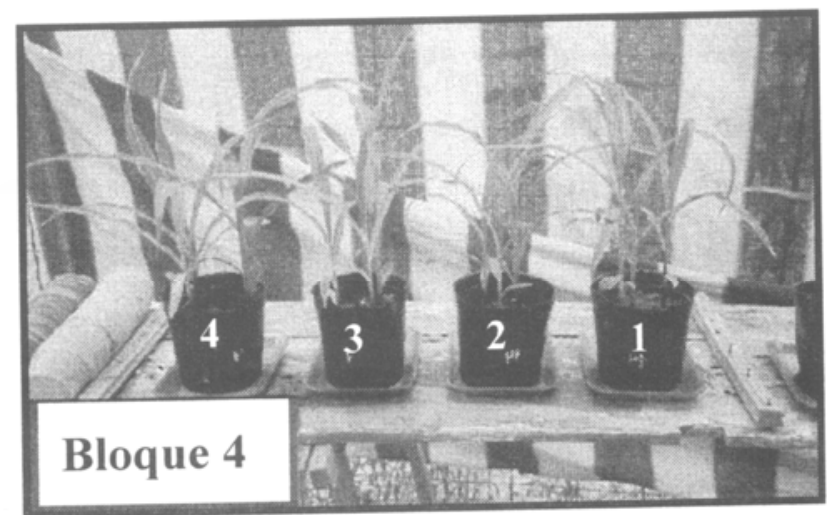

Fotografía 1. Crecimiento de las plantas inoculadas con las diferentes cepas.

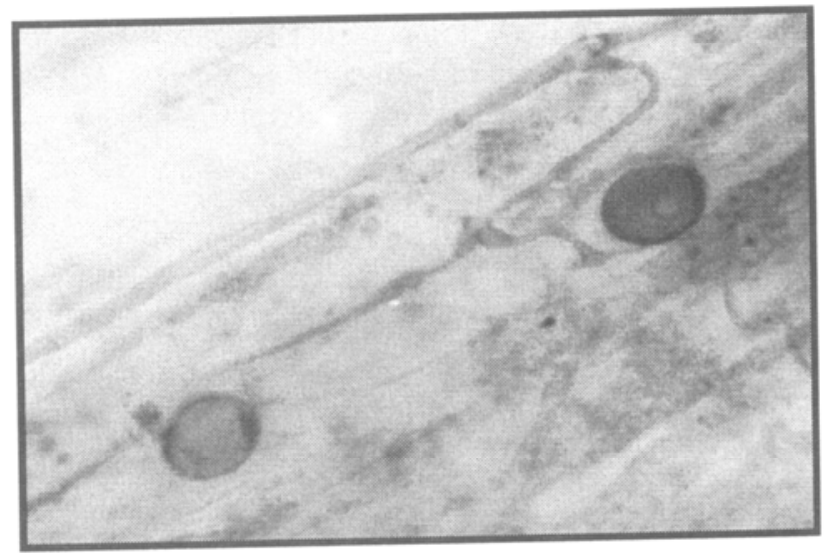

Fotografía 2.- Vista de raíz micorrizada $1000 \mathrm{X}$ -

cando una diferencia del $11 \%$ menos que el testigo, manteniéndose a lo largo de las dos mediciones restantes.

\section{Actividad Amonificante.}

No se registro liberación de amonio en ninguno de los tratamientos realizados, lo que lleva a inferir en una buena nitrificación durante el tiempo de incubación.

Degradación de celulosa.

Del análisis de los datos de la masa remanente en la evaluación de la actividad celulolítica, no se presentaron diferencias significativas. Superando en todos los casaos el $80 \%$ de degradación con solo 21 días de incubación.

\section{Presencia de fijadores libres.}

Para la presencia de Azotobacter, se observó variaciones entre bloques. Para el primer bloque los tratamientos 1,3 y 4 presentan algunas colonias. Para el bloque 3 el tratamiento 4 presentó una colonia. Para el bloque 4 los tratamientos 1,3 y 4 presentaron algunas colo- 
nias en tanto que el tratamiento 2 cubrió toda la superficie. En el bloque 5 el tratamiento 1 y 2 cubrieron toda la superficie en tanto que los tratamientos 3 y 4 presentaron algunas colonias.

\section{CONCLUSION}

Se concluye que la micorrización fue infectiva para los tres inóculos ensayados, destacándose el tratamiento 2- Glomus intraradices Schenck \& Smith (1982) Aislamiento SB1; 3. Ninguno de los tratamientos reali- zados beneficiaron el desarrollo del cultivo en las condiciones de este trabajo.

Según los datos obtenidos se puede decir que la inoculación con micorrizas no afectaría, en primera instancia, la actividad biológica del suelo utilizado.

\section{AGRADECIMIENTOS}

Al Instituto Spegazzini, La Plata, Buenos Aires, Al Dr Orlando Popoff y al Ing. Agr. Camilo Quarín.

\section{REFERENCIAS}

Covacevich, F.; Echeverria, H. E. \& Gianinazzi, S. (2000). XVII Congreso Argentino de la Ciencia del Suelo. Mar del Plata.

Crovetto, C. (2000). "La cero labranza permanente y la disponibilidad de nutrientes para las plantas” $8^{\circ}$ Congreso Nacional de AAPRESID. Mar del Plata, 16 al 19 de Agosto de 2000. Tomo I.

Duchesne, L. (1994). Mycorrhizae and plant health. Symposium series APS PRESS. Minnesota, pp. 27-46

Frioni, L. (1990). Ecología Microbiana del Suelo. Dpto. de Publicaciones y Ediciones de la Universidad de la Republica. Montevideo, Uruguay.

Frioni, L. (1999). Procesos microbianos. Tomos I y II. Ed. de la Fundación de U. N. de Río Cuarto.

Fuentes, P. \& Bermudez, J. (1971). Método para medir la mineralización del carbono en el suelo” Actas de la 6 a Reunión Argentina de la Ciencia del suelo. Córdoba.

Honrubia, M.; Torres, P.; Díaz, G. \& Morte, A. (1995). Biotecnología Forestal: Técnicas de Micorrización y Mocropropagación de plantas. Universidad de Murcia.

Linderman, R. G. (1994). Mycorrhizae and plant health. Symposium series APS PRESS. Minnesota, pp. 1-26
Miller, S. \& Allen, E. (1992). Mycorrhizal Functioning. M F Allen Ed. Chapman \& Hall. 301-332

Nurlaeny, N.; Marschner, H. \& George, E. (1996). Plant and soil 181: 275-285

Page, A.; Miller, R. \& Keeney,E. (Eds). (1982). Methods of soil analysis, part 2. Chemical and microbiological properties. $2^{\text {a }}$ Ed. Madison, Wisconsin, USA.

Primavesi, A. (1984). Manejo ecológico del suelo. $5^{\mathrm{a}}$ ed. El Ateneo, Buenos Aires, Argentina.

Quantz, B.; Bakos, B. \& Iglesias, M. C. (1981). El suelo como sistema viviente. Guía de trabajos prácticos. Cátedra de Microbiología Agrícola. Facultad de Ciencias Agrarias. UNNE.

Schinner, F.; Ohlinger, R.; Jandeler, E. \& Margesin, R. (1996). Methods in soil Biology. E d. Springer Verlag.

Sylvia, D. M. (2000). “Mycorrhizal Symbioses”. On line. Disponible http:/ / www.ifas.ufl.edu/ dmsa/mycorrhiza.htm. 